\title{
Retornantes internos por covid-19: una mirada desde la desigualdad y la informalidad
}

\author{
Internal returnees for covid-19: a look from inequality and informality
}

Teódulo Gerardo Lázaro Aquino' (iD

\author{
Cómo citar \\ Lázaro Aquino, T. G. (2021). Retornantes internos por covid-19: una mirada desde la desigualdad y la informalidad. Socialium, 5(1), \\ 23-36. https://doi.org/10.26490/uncp.sl.2021.5.1.738
}

${ }^{1}$ Doctor en Sociología, Magister en Salud Pública, Licenciado en Sociología, Pontificia Universidad Católica del Perú, Lima, Perú

g.lazaroa@pucp.edu.pe Google Scholar

Arbitrado por pares ciegos Recibido: $13 / 10 / 2020$ Aceptado: $19 / 12 / 20$

\section{RESUMEN}

El estudio tuvo por objetivos: 1) Identificar quiénes fueron los retornantes, 2) Analizar los factores de retorno interno y 3) Explicar tanto las dinámicas como los tipos de retorno. Para ello, se hizo utilizó el método cualitativo de investigación, en su modalidad de diseño narrativo. La principal técnica de recolección de información fue la observación documental (diarios, revistas, webinares, entrevistas en Internet, entre otros). Se concluye en que, si bien hubo elementos coyunturales que favorecieron al fenómeno del retorno como la cuarentena, el aislamiento social, el distanciamiento social y el estado de emergencia, fueron dos los factores estructurales desencadenantes: la desigualdad y la informalidad del país. Asimismo, el desplazamiento de los retornantes por covid-19, una forma de migración forzada, tomaría dos tendencias principales: uno, sería migración de retorno cuando los actores toman la decisión definitiva de quedarse en sus lugares de origen y dos, de reemigración si deciden volver nuevamente a sus destinos de migrantes. Ello depende del tiempo que dure la pandemia, el aislamiento o confinamiento social, el estado de emergencia, pero sobre todo del desenlace que tome la crisis sanitaria, económica y social en curso.

Palabras clave: migración por retorno; retornantes; desigualdad; informalidad.

\section{ABSTRACT}

The study's objectives were: 1) Identify who were the returnees, 2) Analyze the internal return factors and 3) Explain both the dynamics and types of return. For this, the qualitative research method was used, in its narrative design modality. The main information gathering technique was documentary observation (newspapers, magazines, webinars, Internet interviews, among others). It is concluded that, although there were conjunctural elements that favored the phenomenon of return such as quarantine, social isolation, social distancing and the state of emergency, there were two structural triggers: inequality and informality in the country. Likewise, the displacement of returnees due to covid-19, a form of forced migration, would take two main trends: one, it would be return migration when the actors make the final decision to stay in their places of origin and two, of re-migration if they decide return again to their migrant destinations. This depends on the duration of the pandemic, the isolation or social confinement, the state of emergency, but above all on the outcome of the ongoing health, economic and social crisis.

Keywords: migration by return; returnees; inequality; informality. 


\section{Introducción}

La historia humana muestra desplazamientos permanentes de personas o grupos en búsqueda de nuevos lugares de asentamientos. Muchas veces en grandes oleadas. Estas pueden ser voluntarias o forzadas (compulsivas). Depende del motivo que las impulsan. La gran mayoría son por factores económicos (crisis económicas, búsqueda de empleos y nuevos ingresos), políticos (guerras internas o externas), ambientales (huaycos, deslizamientos, terremotos) y la ejecución de políticas públicas (grandes proyectos mineros, hidro energéticos e infraestructura vial).

En el Perú, hubo tres grandes desplazamientos poblacionales. En los años 60 y 70, el denominado "desborde popular" fue básicamente por cuestiones económicas. En los años 80 y principios de los 90, se dio por la violencia política y el terrorismo. Y la última, el 2020, se debió a la crisis económica y el aislamiento social debido a la expansión y consecuencias del covid-19.

El estudio aborda este último proceso, pero solo la modalidad de migración de retorno y de carácter interno: de Lima hacia las regiones o viceversa, así como las movilizaciones intrarregionales. De hecho, se ha obviado el retorno internacional.

Para enrumbar los objetivos y responder a las preguntas de investigación, se tomaron como conceptos guía:

La migración de retorno: Si bien el concepto no presenta límites precisos, su utilidad estaría supeditada solo para aquellas personas que retornan por primera vez a su región o país. Ya que, desde el momento en que los individuos se trasladan a un segundo destino supone una migración de tránsito. En el caso que emigran nuevamente al mismo destino después de haber retornado por primera vez se llama reemigración. Mientras cuando emigran a un nuevo destino tras haber retornado, se está frente a una nueva emigración. Finalmente, cuando los movimientos son de ida y vuelta entre dos lugares, es de decir, incluyen más de un retorno, se trata de una migración circular (Bovenkerk, 1974, citado por Castillo, 1997).

La desigualdad social: Al respecto, en Sociología existen por los menos dos puntos de vista. Según Tilly (2000), las desigualdades se originan en todo tipo de organización de la sociedad. En el entendido que los seres humanos fundan sistemas de desigualdades a través de dos mecanismos causales: 1 ) La explotación: cuando las personas con poder y relación disponen de recursos de los que extraen altas utilidades y sacan provecho del esfuerzo de personas ajenas, a quienes también excluye de todo el valor agregado y 2) El acaparamiento de oportunidades: en el sentido que integrantes de determinadas redes dominan y monopolizan el acceso a recursos valiosos, en desmedro de otros. 
Ambos mecanismos actúan en paralelo, y se apoyan en otros dos: la emulación, que implica la reproducción de modelos organizacionales y/o el traspaso de relaciones sociales de otros ámbitos, y la adaptación que consiste en la implementación de rutinas sobre la base de estructuras desiguales. Así, mientras la explotación y el acaparamiento de oportunidades contribuyen al establecimiento de la desigualdad, la emulación y la adaptación expanden su influencia.

Para Dubet (2015), las desigualdades se originan en las crisis de las solidaridades. Ya que estas descansan en tres ejes: 1) En la interrelación de las actividades sociales y económicas, en la división del trabajo en el que se necesitan unos y otros; 2 ) En el contrato social que, al proceder de un acuerdo político, le otorga dicho carácter; y, 3) En la fraternidad como imaginario de lo simbólico, que se aprende en el sentido común para considerarse "hermanos". Así, la debilidad en estos lazos de solidaridad explica la ampliación de las desigualdades. Pues, para que la igualdad social se convierta en voluntad colectiva, debe consolidarse los sentimientos y las prácticas de fraternidad y solidaridad. En consecuencia, la desigualdad se expresa en la diferencia extrema e injustificada en el acceso y disfrute de recursos y oportunidades para los ciudadanos. Esta puede analizarse en cinco ejes: la justicia tributaria, la inversión en las personas, el empleo digno, los derechos económicos de las mujeres, y la gobernanza socioambiental y de los recursos naturales (Mendoza, 2019).

La informalidad: Se refiere a personas que no cumplen con los compromisos contraídos; o algo que no es oficial o no sigue normas estrictas. Este último, muy cerca del concepto de anomia. Al respecto, señala López (2009), para Emile Durkheim la anomia implica la inexistencia de normas que rigen las relaciones entre las diversas funciones sociales, cada vez son más complejas debido a la división del trabajo y la constante especialización. Las dos situaciones claras de anomia son en el ámbito económico y la situación conyugal. En la economía la anomia se deriva del permanente cambio en los sistemas de producción. Pues, las normas que sirven como factores de organización se debilitan sin ser sustituidas por otras que respondan a los nuevos contextos. Mientras para Robert Merton, la anomia es consecuencia del fraccionamiento de la estructura cultural de la sociedad. Pues, la transformación de la sociedad tradicional a moderna, ha derivado en el desorden de las normas culturales, dando origen a un desfase entre los objetivos "legítimos" de los grupos humanos y los medios para lograrlos, con el rol crucial de las variables socioeconómicas. Finalmente, para Talcott Parsons, la anomia está vinculada al desarreglo producido en las personas como efecto de los períodos económicos cambiantes y los cambios radicales en su medio social. De forma que, en las crisis positivas o negativas, las posibilidades y oportunidades que se puede hacer o lograr varía, 
produciéndose un cambio en la relación medios - fines, dando lugar a la confusión. Y aparece la necesidad de un ajuste en la práctica y el comportamiento de los individuos.

De manera que la informalidad (y la anomia) ha tenido un papel central en el discurso del desarrollo, desde que apareció en la década del 70. Por un lado, su elasticidad conceptual ha permitido la incorporación de una diversidad de interpretaciones suficientemente comunes como para referirse al mismo fenómeno. De allí que la informalidad denota una actividad económica que se encuentra al margen del alcance de la regulación estatal, ya sea porque no se aplican o no se cumplen. Por otro, la informalidad se contextualiza en el enfoque del "desarrollo dual", que diferencia en la economía entre un sector "moderno" y otro "tradicional". Así, la informalidad está relacionada a la pobreza y una actividad económica con baja productividad y baja generación de ingresos. $Y$, finalmente, la informalidad abarca a la situación de todos los empleos en los que no existe un empleador-protector (Kanbur, 2010).

En ese sentido, la informalidad alude a personas pobres, extremos pobres y vulnerables, que sobreviven realizando oficios, actividades y tareas de pequeña escala que, -sin ser actividades delictivas-, están al margen de las normas. Incluye actividades de sobrevivencia y rentables, pero no reconocidas, no registradas, no protegidas y no reguladas. Se trata de trabajadores no cubiertos por la legislación laboral, no sujetos a las reglas formales del trabajo ni a la justicia laboral; que no cotizan a la seguridad social. En su mayoría tienen empleos de baja productividad y bajos ingresos. Así, las empresas como los trabajadores informales forman parte de la fractura social que erosiona la cohesión social (Salazar y Chacaltana, 2018).

\section{Método}

Se hizo uso del método cualitativo de investigación. El diseño específico fue de tipo narrativo, que busca entender hechos, situaciones, procesos, eventos e interacciones de actores (Hernández y Mendoza, 2018), en este caso, relacionados al fenómeno migratorio de retorno por covid-19.

Es decir, se focalizó acontecimientos o eventos de retorno protagonizado por miles de familias en busca de regresar a sus lugares de origen, en busca de lograr un acercamiento objetivo a los actores de la migración de retorno. Como refiere Flick (2015), en la investigación cualitativa, el muestreo no se guía, en la mayoría de casos, por una elección formal de una parte de la población existente o supuesta. También se trata de establecer una selección de casos, materiales o acontecimientos elegidos deliberadamente para construir un corpus teórico y empírico sobre el fenómeno de estudio. 
La técnica principal de recolección de información fue la observación documental, que consistió en la identificación, muestreo y resumen analítico de los acontecimientos de retorno en diarios, artículos de revistas, webinares, videos, informes y declaraciones oficiales de dirigentes del gobierno, así como entrevistas encontradas en Internet. Respecto, al análisis de los datos; primero se elaboró un esquema de presentación de los resultados en base a los objetivos y las preguntas de investigación, luego, se introdujo la información por cada dimensión de estudio, para finalmente, realizar el análisis correspondiente.

\section{Resultado y Discusión}

El contexto nacional de migración 2012-2017: A continuación, de manera concisa y abstracta se describe a la población que potencialmente habría sido la que decidió regresar a sus lugares de origen.

En el Perú, el flujo de personas y familias en desplazamiento continúa siendo permanente y oscilante, hacia distintas regiones y ciudades, especialmente Lima (La Capital). En el período 2012-2017, según el INEI (2020), las regiones más expulsoras fueron: Cajamarca, Loreto, Puno, Huancavelica, Ancash, Piura, Junín, Huánuco, Cusco, Lambayeque, Ayacucho, Amazonas, Pasco, Apurímac y San Martín. Y las más atrayentes: Lima (de lejos), Arequipa, Tacna, Ica, Callao, Madre de Dios, Ucayali, Moquegua, La libertad y Tumbes. Los migrantes recientes llegados a Lima, en su mayoría, fueron jóvenes entre 20 y 24 años de hombres y mujeres, seguido de los grupos de 15 a 19 y 25 a 29 años de edad. Lo que coincide con la edad de estudio y trabajo. El nivel educativo alcanzado era básicamente secundario $(46,2 \%)$. Quienes tenían educación superior no universitaria y universitaria, significaron el 17,4\% y $23,9 \%$, respectivamente. Mientras la pobreza monetaria afectaba al $26,8 \%$ de ellos. Siendo la búsqueda de empleo la principal razón de migración. Asimismo, el 21,4\% de la población ocupada migrante se dedicaba al Comercio; el 13,4\% trabajaba en actividades de Manufactura; el 8,9\% en Transportes y Comunicaciones y el $44,2 \%$ realizaba Otros Servicios. Además, el $69,0 \%$ eran trabajadores dependientes o asalariados (empleado u obrero) y casi la cuarta parte $(24,7 \%)$ trabajadores independientes (empleador o patrono e independiente o cuenta propia). En su mayoría vivían en viviendas alquiladas. Sin embargo, muchos accedieron a una vivienda propia, por invasión. En su mayoría, ubicadas en Asentamientos Humanos, y el $24,5 \%$ sin título de propiedad. Sumado a la carencia o limitado acceso a los servicios básicos en los lugares de llegada.

Identificando a los retornantes internos: Los acontecimientos de desplazamiento y retorno interno forzado por el contexto del covid-19, suscitado de Lima hacia las regiones y viceversa, y entre las 
mismas regiones, tuvo como actores principales a cinco tipos de personas: a) Un grupo compuesto por personas que se habían trasladado a Lima por razones médicas (citas o tratamiento) o creyeron que aquí obtendrían mejores opciones de salud que en sus regiones; b) Otro sector fue trabajadores eventuales o temporales que llegaron a Lima, durante los tres meses de verano, que luego regresan a su residencia habitual en las regiones; c) Un tercero fueron familias, donde un miembro había conseguido trabajo, ya sea en Lima o en la capital de la región, que había trasladado a su familia y convivían en habitaciones, muchas en un solo cuarto. Se trataba de alguien que laboraba de alguna manera formal como chofer o vendedor, pero los demás miembros eran informales. Sin embargo, la familia estaba completa; d) Un cuarto agrupamiento eran personas que vivían bastante tiempo en Lima, en condición de informales o trabajos eventuales. Pero, a raíz del aislamiento social se quedaron sin trabajo, es decir, sin medios de subsistencia; e) El quinto colectivo fueron personas con ciertas condiciones de vida en Lima, como techo, alimentación o viven en una casa, de su propiedad o de un pariente. Pero a pesar de tener condiciones de subsistencia decidieron retornar a su región convencidos de que tendría mejores oportunidades (Muñoz, 2020) de enfrentar la situación de crisis económica y la pandemia.

Para Coral (2020), un primer grupo de retornantes estuvo integrado, por un lado, por personas o familias que llegaron a las ciudades por distintos motivos. Al verse sorprendidos por la cuarentena quedaron varados, pero serían los menos. Y por otra, por hijos, nietos y demás familiares, de las víctimas del conflicto armado. Muchos de ellos nacidos en las ciudades. Se calcula la existencia de un aproximado de 2 mil asentamientos de desplazados a nivel nacional. Un segundo grupo serían migrantes recientes de las últimas décadas, que habrían venido por voluntad propia y con expectativas de desarrollo, presionados y condicionados, a la vez, por factores de riesgo económicos y sociales. Muchas familias estuvieron en las ciudades en condición de marginalidad, pobreza extrema y exclusión social, no pudiendo garantizar ni su sobrevivencia. Por lo tanto, se trata de un retorno forzado.

Asimismo, entre los retornantes estarían estudiantes de colegios, institutos, universidades, incluso postulantes que estaban en ciudades distintas a la de su origen, que ante las nuevas condiciones de educación a distancia por internet no tenían razón para estar lejos de su familia generando gastos de alojamiento y alimentación. Así como personas y familias en búsqueda de refugio de largo plazo, principalmente en ciudades intermedias o semirurales, donde tendrían más oportunidades y mejores condiciones de vida que en los precarios asentamientos humanos de Lima y otras ciudades, donde vivían hacinados, sin servicios básicos y con muchos costos para generarse ingresos o abastecerse en condiciones de restricción de la movilidad. Se trataría de una migración hacia ciudades intermedias 
de la sierra y la selva, no sólo huyendo de la pandemia, sino en búsqueda de oportunidades de supervivencia y de trabajo que no van a conseguir en Lima, por las eventuales restricciones a la generación informal de ingresos, la crisis laboral y la recesión económica (Coral, 2020).

Finalmente, según Rosel (2020), la migración de retorno distingue, por un lado, a migrantes temporales sin arraigo que ocurren en los meses de verano. Siendo un típico caso el heladero que recorre en las playas. Por otro, a personas que, por razones de abastecimiento, comercio, salud, trabajo temporal estuvieron en ciudades que no son de su residencia habitual. $\mathrm{Y}$, por último, a quienes buscaban regresar para atender a sus familiares que requerían ayuda, especialmente las personas mayores.

Factores causales de retorno: El desplazamiento forzado no reaparece con la pandemia. Siempre estuvo presente, como procesos inconclusos de la violencia política o nuevos procesos inducidos por factores expulsores diversos. Como el caso de cinco comunidades hostigadas y expulsadas del Valle de los ríos Apurímac, Ene y Mantaro (VRAEM) por el narcotráfico; las comunidades desplazadas por la expansión de empresas mineras como Fuerobamba y Toromocho; y, muchas familias desplazadas del norte del país debido al fenómeno del niño. La pandemia solo fue el detonante de la crisis (Coral, 2020) y el desplazamiento interno.

En ese sentido, las personas o familias decidieron retornar al perder su empleo (como albañiles de obras, en comercio, manufactura, etc.), cuando no tenían cómo pagar el alquiler de su habitación o cómo mantener a su familia o a sí mismo, y optaron por recurrir a su región de origen, como una apuesta para reducir las probabilidades de sufrir hambre, antes que por el temor al propio virus. Es decir, retornaron por la falta de recursos para subsistir. Y la salida inmediata fue buscar refugio en los pueblos originarios, donde habría mayores recursos de sobrevivencia (Pecho, 2020).

Es una situación que visibiliza las vulnerabilidades de la población en una economía mayoritariamente informal, precaria, sin derechos, que se agrieta por sus lados más frágiles (Defensoría del Pueblo, 2020a), que son las personas y familias que no lograron insertarse o integrarse a las ciudades a donde migraron en busca de mayores y mejores oportunidades de desarrollo personal o familiar. En todo caso, se trata de una inserción o integración informal o parcial, sin mayores derechos. Condición que lograrían las siguientes generaciones de las familias migrantes, básicamente a partir del factor educación.

Como testimonia Elizabeth: Ella había llegado a Lima hace dos años, con el fin de encontrar trabajo. Hasta antes de la pandemia vendía frutas en su triciclo recorriendo las calles. Esto cambió el domingo 15 de marzo, cuando se declaró el Estado de Emergencia por el coronavirus. Salió de El Agustino, 
donde alquilaba un minidepartamento junto a su familia. Pues, se había agotado el poco ahorro que tenía y dejó la casa alquilada, donde vivía con su familia. Y presionada por la falta de recursos económicos, cogió a los suyos, llenó unos maletines de ropa e inicio su viaje a pie por la Carretera Central. El 15 de abril decidieron dejar Lima y comenzaron la caminata (Zapata et al, 2020).

Sin embargo, estos factores son coyunturales. Existen factores estructurales, más profundos, como la desigualdad y la informalidad, que explican las razones de la migración de retorno de las personas y familias identificadas. Ya que la pandemia y la cuarentena pusieron en evidencia la profunda precariedad y desigualdad del sistema social y la estructura económica del país.

Como señala Mendoza (2017 y 2019), el Perú es un país altamente desigualdad, con barreras y diferencias extremas e injustificadas que separan a sus habitantes, evidenciadas en distintas formas y ámbitos: como el acceso diferenciado a una educación de calidad; los salarios inequitativos entre hombres y mujeres; los precarios sistemas de servicios de salud, con amplia subcobertura, entre otros, que limitan el bienestar y las oportunidades de desarrollo de las personas. El crecimiento económico (2005-2012) estuvo marcado por la precariedad y la desigualdad que se constituyeron en impedimentos para la ampliación de las capacidades de las familias, que no pudieron resolver sus condiciones de dependencia, vulnerabilidad y exclusión en que han sobrevivido.

Agrega OXFAM (2015), en el Perú dependiendo de dónde se nace y vive las diferencias en calidad de vida, en acceso a servicios y oportunidades son tan inmorales. En Lima, el nivel de escolaridad en los adultos es casi 11 años, duplica a Huancavelica (5.5 años); la tasa de mortalidad infantil de Lima es 14 por mil nacidos vivos, triplica a Loreto (40 por mil nacidos vivos); la tasa de desnutrición crónica en la niñez en Huancavelica es 35\%, 10 veces mayor que la de Tacna (3.7\%).

Para tener una idea sobre la desigualdad de ingresos, se muestra la figura siguiente. Menciona OXFAM (2015), las brechas entre quienes cuentan con recursos y oportunidades y quienes no, se reflejan en instrumentos de análisis como el Índice de Gini, que se utiliza para medir la desigualdad en la distribución del ingreso. En el caso peruano, el valor elevado del Gini refleja la profundidad de la desigualdad. Si bien hubo una reducción moderada del índice desde mediados de la pasada década, los niveles son altos y en los últimos años se estancó la reducción. Factores como la desaceleración económica y el retroceso fiscal, podrían revertir los modestos avances logrados. 


\section{Figura 1}

La desigualdad de ingreso en el Perú

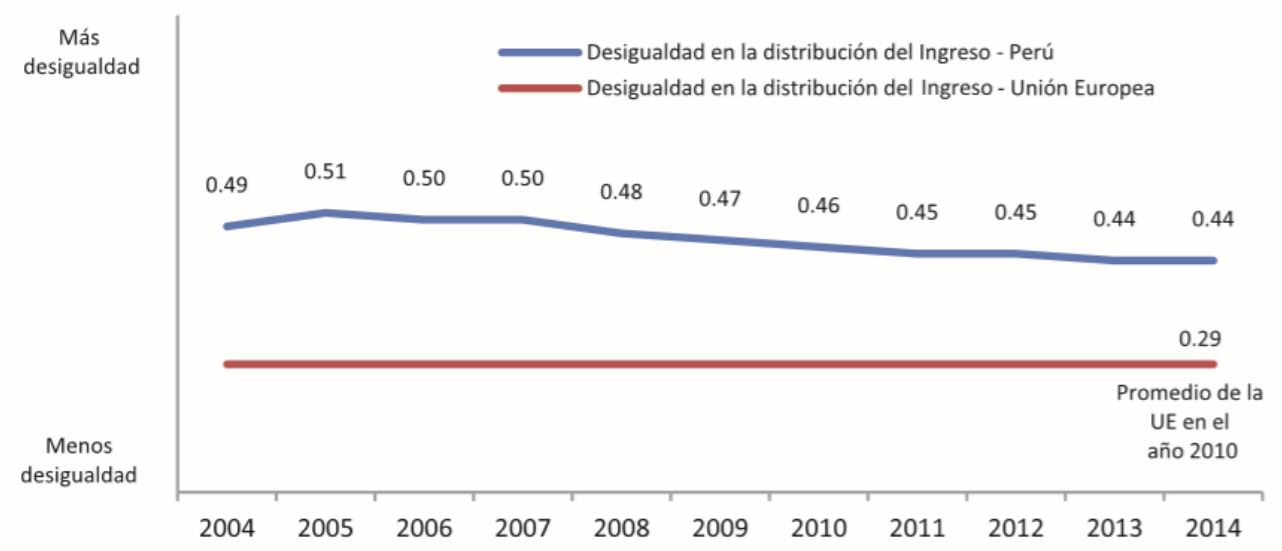

Nota. Tomado de OXFAM, 2015.

En efecto, en el contexto de la pandemia la desigualdad ha aumentado. Así lo refleja una encuesta del Banco Mundial (2020), cuyos resultados muestran, por un lado, la pérdida de empleo; por otro, la diferencia en la preservación del empleo según la condición de trabajador asalariado y no asalariado, el nivel de educación alcanzado, la condición de género (ventaja del hombre sobre la mujer) o la competencia en teletrabajo; asimismo, la reducción muy alta de los ingresos familiares. Seguido de la inseguridad alimentaria y falta de acceso a servicios médicos, focalizado en las personas adultas. Y la falta de conectividad de los hogares más vulnerables, siendo una barrera importante en el acceso a la educación en todos los niveles.

A esta realidad, se agrega la situación de informalidad en el país. Al finalizar el año 2019, la población ocupada con empleo informal alcanzó el 72,7\%. Es decir, 12’462,800 personas tenían empleos no sujetos a la legislación laboral nacional, no pertenecían al sector formal de la economía. La región Huancavelica registró la tasa más alta de informalidad laboral (92\%); seguida de Cajamarca (87,9\%), Puno $(87,6 \%)$, Ayacucho $(87,3 \%)$ y Huánuco $(86,6 \%)$. Ica registró la más baja $(61,4 \%)$; seguida de Moquegua $(65,4 \%)$, Arequipa $(66,2 \%)$, Lima $(60,2 \%)$ y Callao $(57,8 \%)$. Según sectores económicos: la agricultura, pesca y minería, que concentraban a 4’065,000 personas, presenta una tasa de informalidad de 93,6\%; mientras hoteles y restaurantes, que albergaba a 1'073,100 personas, 
registró una tasa de 84,8\%; en tanto transportes y comunicaciones, que concentraba a 1'169,200 personas, tuvo una tasa de 81,3\% (PERUCÁMARAS, 2020).

Como puede verse, las condiciones de desigualdad e informalidad en el país y las regiones, marcan las decisiones que toman las familias respecto a su vida futura y cotidiana.

Dinámica de retorno y cantidad de retornantes: La migración de retorno por covid-19, presenta dos dinámicas formas principales: de Lima hacia las regiones, que fue la más invisible y viceversa; y otra interregional e intrarregional, la menos visible o invisibilizada. Esta última, es la migración que no se ve, porque no ocurre en Lima. Siendo igual o más dramática. Ocurre entre regiones. Gente de Ayacucho, Arequipa, Juliaca estuvieron en las capitales buscando volver a su pueblo. En Cusco, personas que trabajaban en Turismo (como mozos, choferes, cocineros de restaurantes) se quedaron en la calle y volvieron a las provincias de Cusco (Rosel, 2020). En el caso de las salidas de Lima hacia las regiones, se concentraron en cinco puntos: Carretera Central, La Victoria, Panamericana Norte, Chilca y Lurín (Zapata et al, 2020).

Es decir, la crisis de retorno se originó en las ciudades, especialmente en Lima, y se expandió a otras regiones, involucrando a ciudades capitales de provincias y distritos al interior de las regiones. Fue un proceso defensivo frente al hambre y el miedo a la enfermedad y la muerte, en busca inmediata de un punto de apoyo para acumular fuerzas y revertir la situación difícil. Es una migración de retorno, pues el destino final son las comunidades de origen. Sin embargo, es improbable que dejen de forma definitiva las ciudades. Superado el momento crítico, con seguridad habrá un proceso de reacomodo, en la lógica de la "estrategia de los dos pies", a fin de mantener los dos espacios (Coral, 2020).

Por otro lado, es difícil saber con exactitud la cantidad de retornantes, porque tomaron proporciones inesperadas y una dinámica compleja. Muchos retornos fueron "formales", en alguna forma organizadas desde el gobierno central y los gobiernos regionales, a través de los llamados "viajes y vuelos humanitarios". Pero otros lo hicieron de manera clandestina.

Así, desde el gobierno central, se habló de alrededor de 220,000 personas empadronadas, solo para retornar de Lima a su lugar de origen, de manera ordenada y gradual (Muñoz, 2020). 
El proceso de retorno muestra que, hasta el primero de abril, habrían salido de Lima 4 mil personas ${ }^{1}$. Hacia el 4 de mayo, se habría trasladado a 18,020 personas². Al 16 de mayo, serían 24,606 personas trasladadas. De ellas, 17803 de Lima hacia las regiones, 4716 de las regiones hacia Lima y 2087 entre regiones. Mientras al 22 de junio, habría aumentado el número a 43117 personas. Por vía terrestre (34 171) y vía aérea (8 946) (Defensoría del Pueblo, 2020b).

\section{Conclusión}

Como puede verse, los cuatro factores coyunturales principales de la migración de retorno fueron: la cuarentena $^{3}$, el aislamiento social ${ }^{4}$, el distanciamiento social ${ }^{5}$ y el estado de emergencia ${ }^{6}$. Que transformaron radicalmente el medio social de convivencia y las condiciones de subsistencia de los migrantes y sus familias. Es decir, no fueron barreras de contención frente a la propagación del covid19, a través de la inmovilización de las personas. Al contrario, de inmediato activaron, no las alarmas, sino los resortes de inserción urbana informal que habían logrado construir. Una de ellas, la más importante, fueron las escasas fuentes de ingresos que las sostenían, que colapsaron ante la suspensión de las relaciones económicas, cuyas consecuencias mediatas fueron la crisis alimentaria familiar, la falta de capacidad de pago de las habitaciones donde convivían, entre otros.

Esta situación alimentó la decisión de retornar a los lugares de origen, como alternativa ante la crisis de sobrevivencia. $Y$ ante la paralización de todos los medios de transporte, optaron por caminar. De allí el mote de "caminantes", que hicieron visible los medios de comunicación, por el tamaño de la

\footnotetext{
${ }^{1}$ Defensa Civil apoya salida de 4000 peruanos desde Lima a sus regiones, por el covid-19. (21 de abril de 2020). El Comercio. URL https://www.elcomercio.com/actualidad/peru-salida-personas-lima-coronavirus.html.

2 PCM coordinó el traslado de más de 18,000 personas a sus regiones. (4 de mayo de 2020). Andina. URL https://andina.pe/agencia/noticia-pcm-coordino-traslado-mas-18000-personas-a-sus-regiones-795810.aspx.

${ }^{3}$ Cuarentena: Consiste en separar a alguien de los demás, completamente asintomático, por la eventualidad de que llegue a presentar síntomas de la enfermedad. Ver: https://newsnetwork.mayoclinic.org/discussion/covid19-cual-es-la-diferencia-entre-cuarentena-y-aislamiento/.

${ }^{4}$ Aislamiento: Consiste en separar del resto a una persona con resultado positivo para la enfermedad y mantenerla aislada. Ver: https://newsnetwork.mayoclinic.org/discussion/covid-19-cual-es-la-diferencia-entre-cuarentena-yaislamiento/. Aislamiento social obligatorio: medida del estado de emergencia por coronavirus para evitar el tránsito de personas por las vías públicas. Ver: https://www.gob.pe/8784-coronavirus-preguntas-y-respuestassobre-el-estado-de-emergencia. Inmovilización social obligatoria: medida más restrictiva que el aislamiento social que exige permanecer en casa en determinadas horas del día. Ver: https://www.gob.pe/8784-coronaviruspreguntas-y-respuestas-sobre-el-estado-de-emergencia.

${ }^{5}$ Distanciamiento social: También llamado "distanciamiento físico", consiste en mantener una distancia segura entre una persona y otras que no pertenecen a su hogar. Ver: https://espanol.cdc.gov/coronavirus/2019ncov/prevent-getting-sick/social-distancing.html.

${ }^{6}$ Estado de emergencia: Restricción de derechos como reunión con otros ciudadanos y libre tránsito dentro y fuera del territorio peruano. Ver: https://www.gob.pe/8784-coronavirus-preguntas-y-respuestas-sobre-el-estado-deemergencia
} 
concentración de retornantes, que tomaron, como en el caso de Lima, las carreteras principales para enrumbar a sus regiones.

El retorno se complicó por cinco motivos: 1) El riesgo de propagación del covid-19, por eso, se hicieron las "pruebas rápidas"; 2) La falta de medios de transporte, que cumplan con los protocolos de seguridad; 3) La magnitud de personas y familias en desplazamiento, que superaron la capacidad de apoyo, tanto del gobierno central y de los gobiernos regionales, que se comprometieron para el traslado; 4) La logística itinerante para su traslado hasta sus lugares de origen, habitaciones aisladas, comida, seguridad, etc., ya que tuvieron que permanecer en cuarentena durante 14 días, para recién continuar hacia su destino; $y, 5$ ) La posición adoptada por la población tanto en las rutas de traslado como en los lugares de retorno, ante el temor de contagio.

Finalmente, el desplazamiento de los retornantes por covid-19, tendría tres formas principales. Depende de la alternativa que tomen, después de la crisis sanitaria, económica y social en curso. Si toman la decisión definitiva de no regresar a sus anteriores destinos (como Lima u otras ciudades), sería migración de retorno. Si optan por volver nuevamente a sus destinos, sería una reemigración. Pero si prefieren movilizarse de ida y vuelta entre dos lugares, vale decir, más de un retorno, se estaría frente a una migración circular. Mientras tanto la desigualdad y la informalidad seguirán tallando la vida de los migrantes, de cualquier modalidad, sino se cambian el modelo de desarrollo y económico, impuesto por los grupos de poder dominante bajo el paraguas del enfoque neoliberal.

\section{Referencias}

Banco Mundial (2020). Crisis por el coronavirus aumentó las desigualdades en el Perú. https://www.bancomundial.org/es/news/press-release/2020/09/08/crisis-por-elcoronavirus-aumento-las-desigualdades-en-el-peru.

Castillo, J. (1997). Teorías de la migración de retorno. En: A. Izquierdo y G. Álvarez (coord.) Políticas de retorno de emigrantes. Universidades Coruña. Pp. 29-44.

Coral, I. (2020). Desplazamiento interno por impacto del coronavirus en el Perú. http://www.otramirada.pe/desplazamiento-interno-por-impacto-del-coronavirus-en-elper\%C3\%BA.

Defensoría del Pueblo (2020a). Prevención y gestión de conflictos sociales en el contexto de la pandemia por el Covid-19. Serie Informes Especiales N 026-2020-DP. Defensoría del Pueblo. 
Defensoría del Pueblo (2020b). Migración interna y varados durante la pandemia. acciones desarrolladas por la Defensoría del Pueblo. Serie Informes Especiales № 23-2020-DP. Defensoría del Pueblo.

Dubet, F. (2015). ¿Por qué preferimos la desigualdad? (aunque digamos lo contrario). 1ạ ed. Siglo Veintiuno Editores.

Flick, U. (2015). El diseño de la investigación cualitativa. Ediciones Morata.

Hernández, R. y Mendoza, C. (2018). Metodología de la investigación. Las rutas cuantitativa, cualitativa y mixta. McGraw-Hill Interamericana Editores.

INEI (2020). Estado de la población peruana 2020. Lima: INEI-UNFPA.

Kanbur, R. (2010). Avoiding Informality Traps. http://www.wiego.org/sites/default/files/publications/files/Kanbur_Avoiding.Informality.Tr aps_.pdf.

López, M. (2009). El concepto de anomia de Durkheim y las aportaciones teóricas posteriores. Iberóforum. Revista de Ciencias Sociales de la Universidad Iberoamericana, 8, 130-147.

Mendoza, A. (2017). Brechas latentes índice de avance contra la desigualdad - Perú 2016. OXFAM.

Mendoza, A. (2019). Brechas latentes índice de avance contra la desigualdad - Perú 2017-2018. OXFAM.

Muñoz, F. (2020). Webinar: Movilidad interna en tiempos de Covid-19. IDEHPUCP PUCP, 8 mayo. https://www.youtube.com/watch?v=vPjsaO3_j3w.

OXFAM (2015). Para no retroceder. Realidad y riesgo de la desigualdad en el Perú. Documento de trabajo para Perú № 1. OXFAM.

Pereda, D. (28 de abril de 2020). La mitad teme más al hambre que al coronavirus. Diario La República. https://larepublica.pe/sociedad/2020/04/28/coronavirus-en-peru-encuestanacional-del-iep-revela-que-la-mitad-de-los-peruanos-teme-mas-al-hambre-que-al-covid19/

PERUCAMARAS (2020). El 72,7\% de trabajadores en el país son informales. PERUCAMARAS, edición virtual centro, número 339, 12. 
Rosel, O. (2020). Estamos ante un desborde popular inverso. Una entrevista con Mario Zolezzi. 24 de abril. http://www.desco.org.pe/estamos-ante-un-desborde-popular-inverso-unaentrevista-con-mario-zolezzi.

Salazar, J. y Chacaltana, J. (2018). La informalidad en América Latina y el Caribe: ¿Por qué persiste y cómo superarla? En: J. Salazar y J. Chacaltana (Editores): Políticas de Formalización en América Latina: Avances y Desafíos. OIT. Pp. 13-48.

Tilly, Ch. (2000). La desigualdad persistente. Ediciones Manantial.

Zapata R. et al (2020). La dura travesía de los más pobres: pandemia y desempleo expulsan a miles de migrantes. 25 de abril. https://ojo-publico.com/1786/desplazados-por-la-pandemia-latravesia-de-los-mas-pobres.

\section{Fuentes de financiamiento.}

Autofinanciado.

\section{Conflictos de interés}

No presenta conflicto de intereses.

\section{Correspondencia}

g.lazaroa@pucp.edu.pe. 